\title{
The importance of PSA-Density in active surveillance for prostate cancer
}

\author{
Caner Ediz, Serkan Akan, Muhammed Cihan Temel, Omer Yilmaz \\ Department of Urology, Sultan Abdulhamid Han Education and Research Hospital, Istanbul, Turkey.
}

\begin{abstract}
Summary Objective: In this study, we aimed to determine the predictive factor for additional treatment requirement in active surveillance (AS) for patients with low or very low-risk prostate cancer (PCa) and we investigated the effect of tumor burden by total core involvement rate in biopsy to predict of need for additional treatment. Material and methods: 107 patients with PCa in AS between 2005 and 2018 have been evaluated retrospectively. Groups were divided into two groups according to the need for additional treatment. Group 1 received additional treatment, group 2 did not receive additional treatments and active surveillance was continued. Patient's total prostate-specific antigen (tPSA), prostate-specific antigen density (PSA-D), total core involvement count, quantity and rate at biopsy pathology results and follow-up period were recorded and compared in the two groups.

Results: The current cohort includes 107 patients. Mean age at diagnosis was 63.01years. Mean tPSA values at diagnosis were $6.09 \mathrm{ng} / \mathrm{mL}$ and $5.2 \mathrm{ng} / \mathrm{mL}$ in the group 1 and group 2, respectively. Mean follow-up period was 38.1 months (range, 12 to 134 months). Only PSA-D measurement significantly predicted need for additional treatment $(p=0.017)$. ROC analysis showed that the optimal threshold was $0.13 \mathrm{ng} / \mathrm{mL} / \mathrm{cc}$ (sensitivity: $70.8 \%$; specificity: $57.1 \%$ ). Additional treatment requirement was not detected in patients with PSA-D cut-off level less than $0.07 \mathrm{ng} / \mathrm{mL} / \mathrm{cc}$.

Conclusions: Total tumor burden of less than 5\% is safe for patients with low or very low-risk PCa in AS. A $0.13 \mathrm{ng} / \mathrm{mL} / \mathrm{cc}$ cut-off level of PSA-D can predict to need for additional treatment in patients managed by AS.
\end{abstract}

KEY WORDS: Prostate cancer; Active surveillance; Prostate specific antigen density; Definitive treatment.

Submitted 12 December 2019;Accepted 3 January 2020

\section{INTRODUCTION}

Prostate cancer ( $\mathrm{PCa}$ ) is the most common cancer in men (1) and has a high mortality rate like lung cancer. PSA screening and early diagnosis have led to a decrease in the mortality rates (2). There are many changes in the treatment of prostate cancer over the years and one of the most significant changes is active surveillance (AS) protocol. The time from the diagnosis of low-risk disease to the clinical progression is generally long and progression signs are detectable during the follow-up period. For this reason, AS is applied at low-risk PCa because definitive treatment can be offered when needed. Another reason for choosing AS, is to prevent overtreatment by selecting patients with low-risk prostate cancer in order to preclude possible side effects of the definitive treatment. In 2003, this point was emphasized to avoid or delay definitive treatment and its associated morbidity without compromising survival (35). AS provides these benefits to patients and it is extremely cost effective compared to definitive treatment (6).

Active surveillance has been used for many years in the follow-up of PCa. Unfortunately, we do not know the answer to questions such as "which patients will need additional treatment?" or "when the definitive treatment should be started?" We aimed to evaluate whether total core involvement count, quantity and rate were the correct parameter and we analyzed the efficacy of prostate-specific antigen density or total core involvement rate to predict tumor burden and possible additional treatment needs. We reported outcomes of AS in patients with very low and low-risk PCa.

\section{Materials AND METHOdS}

Study population and design

1695 patients with prostate biopsy performed due to high prostate-specific antigen (PSA) or significant digital rectal examination findings in our clinics between June 2005 and June 2018 were enrolled. The data were collected retrospectively. No ethical committee approval was required owing to the retrospective nature of our study. A total of 117 patients with PCa were managed by AS. The current cohort with available data includes 107 patients. Patient's age, digital rectal examination (DRE) findings, prostate volumes (PV), total PSA (tPSA), PSADensity (PSA-D), in biopsy; total core involvement count (TCIC), quantity (TCIQ) and rate (TCIR), pathology results and follow-up period were recorded.

The criteria for inclusion in the study was as follows: patients with the low risk of cancer progression: > ten years life expectancy, cTl/2, PSA $\leq 10 \mathrm{ng} / \mathrm{mL}$, biopsy Gleason score of $</=6, \leq 2$ positive biopsies, minimal biopsy core involvement ( $\leq 50 \%$ cancer per biopsy).

The criteria for exclusion in the study were: patients who started follow-up before 2003 and patients whose data could not be reached. Patients who voluntarily opted to leave the active surveillance protocol were not included in this study.

The patient's medical records were reviewed. Patients' age, grading and findings of digital rectal examination, 
prostate volumes (PVs) calculated by transrectal ultrasound (TRUS) with the ellipse method (length X depth X width $\mathrm{X} \pi / 6$ ) by using height obtained by transaxial scanning, tPSA, PSA-D that was calculated as tPSA (ng/mL) divided by PV, were eveluated in all patients. In each biopsy. TCIC, TCIQ and TCIR were calculated by total core number (n), total tumor length in all cores $(\mathrm{mm})$ and ratio of total tumor length to total length of biopsy tissues detected within the tumor (\%), respectively. Recurrent pathology results and follow-up time were eveluated. Pathological specimens were reviewed by a single genitourinary pathologist based on the 2005 and 2014 International Society of Urological Pathology (ISUP) Consensus Conference grading of prostate cancer $(7,8)$. The patients who were diagnosed with prostate cancer before 2014 were evaluated using 2005 ISUP grading criteria and patients who were followed-up after 2014 were classified using the 2014 ISUP new grading system. The follow-up protocol included a minimum of four reexaminations per year. tPSA measurement was performed every 3 months for 2 years and then every 6 months in suitable patients. DRE were routinely performed in all patients. Possible tPSA changes were confirmed at least two times. A confirmation biopsy was recommended for all patients within the first year after diagnosis.

Confirmation biopsies generally involve more core assessment than standard biopsies. Multiparametric magnetic resonance imaging of the prostate (mpMRI) was performed in patients who do not want to have a second biopsy or before recurrent biopsies. Patient-based modalities are preferred in the definitive treatment decision. Definitve treatment was planned primarily in patients who were found to be unsuitable for AS. However, androgen deprivation treatment was prefered in patients who did not accept surgical treatment or radiotherapy.

\section{Statistical analysis}

In the analysis of the data, PSPP and Microsoft Excel computer programs were used. As a statistical method in the analysis of data in the research, descriptive analyzes (mean and standard deviation) were applied. In the analysis of the data, the normality hypothesis was first investigated using the Kolmogorov-Smirnov test and Mann Whitney U test was used for data analysis after the normal distribution test. The statistically significant parameters were analyzed by the Receiver Operating Characteristic (ROC) that allowed for the determination of the cutoff value by the combination of the greater specificity and sensitivity by the definition of the area under the curve. The results were evaluated at $95 \%$ confidence interval and $\mathrm{p}<0.05$ significance level.

\section{Table 4.}

\section{RESULTS}

For the 107 patients included in analyses, the mean age at diagnosis was 63.01 years (range, 47 to 74 years). The distributions of all patients by age, PV, tPSA and PSA-D were shown in Table 1 and by TCIC, TCIQ and TCIR in Table 2. An abnormal DRE findings were found in 32 of 107 patients (such as asymmetric growth of lateral lobes, nodule or stiffness). Mean follow up period was 38.1

\section{Table 1.}

Evaluation of descriptive characteristics in patients with PCa were managed by AS.

\begin{tabular}{|l|c|}
\hline Variable & Mean \pm SD (min-max) \\
\hline Age (years) & $63.01 \pm 5.85(47-74)$ \\
\hline Prostate Volume (ml) & $43.47 \pm 17.92(10-114)$ \\
\hline tPSA (ng/mL) & $5.35 \pm 1.96(1.24-10)$ \\
\hline PSA density (ng/mL/cc) & $0.14 \pm 0.09(0.02-0.83)$ \\
\hline SD: standard deviation. AS: active surveillance. & \\
\hline
\end{tabular}

Table 2.

Total core involvement count. quantity and rate of patients according to number of biopsy procedures.

\begin{tabular}{|l|c|c|c|}
\hline & $\begin{array}{c}\text { First biopsy } \mathbf{n}=\mathbf{1 0 7} \\
\text { Mean } \pm \text { SD }(\mathbf{m i n}-\mathbf{m a x})\end{array}$ & $\begin{array}{c}\text { Second biopsy } \mathbf{n}=\mathbf{6 8} \\
\text { Mean } \pm \mathbf{S D} \text { (min-max) }\end{array}$ & $\begin{array}{c}\text { Third biopsy } \mathbf{n}=\mathbf{2 3} \\
\text { Mean } \pm \mathbf{S D} \text { (min-max) }\end{array}$ \\
\hline TCIC (n) & $1.18 \pm 0.39(1-2)$ & $2.2 \pm 1.06(1-5)$ & $2.3 \pm 1.84(1-7)$ \\
\hline TCIQ (mm) & $1.89 \pm 1.25(0.1-7)$ & $5.31 \pm 4.22(1-18)$ & $6.31 \pm 7.2(1-20)$ \\
\hline TCIR (\%) & $14.78 \pm 11.24(0.55-50)$ & $26.23 \pm 19.19(2.5-90)$ & $20.59 \pm 25.8(1-85.8)$ \\
\hline SD: standard deviation. TCIC: Total Core Involvement Count. TClQ: Total Core Involvement Quantity. TCIR: Total Core Involvement Rate. \\
\hline
\end{tabular}

\section{Table 3.}

5-year average of PCa patients who underwent PSA follow-up with active surveillance.

\begin{tabular}{|l|c|c|}
\hline Follow-up period & $\mathbf{n}$ (Overall) & tPSA (ng/mL) (min-max) \\
\hline First year (initial) & 107 & $5.35(1.24-10)$ \\
\hline Second year (confirmation) & 100 & $5.88(0.43-28.1)$ \\
\hline Third year & 69 & $5.86(1.04-12.2)$ \\
\hline Fourth year & 48 & $6.88(0.84-22)$ \\
\hline Fifth year & 32 & $6.56(1.64-19.08)$ \\
\hline
\end{tabular}

Evaluation of descriptive characteristics according to the patients with or without additional treatment requirement (surgical or medical).

\begin{tabular}{|c|c|c|c|}
\hline & $\begin{array}{l}\text { Additional treatment }(-) n=24 \\
\text { Mean } \pm \text { SD (min-max) }\end{array}$ & $\begin{array}{l}\text { Additional treatment }(+) n=83 \\
\text { Mean } \pm S D(\operatorname{Sin}-\max )\end{array}$ & $p$ \\
\hline Age (years) & $63.92 \pm 5.79(57-74)$ & $62.76 \pm 5.84(47-74)$ & 0.74 \\
\hline Prostate Volume (ml) & $40.5 \pm 12.16(15-70)$ & $43.95 \pm 19.45(10-114)$ & 0.72 \\
\hline tPSA (ng/mL) & $6.09 \pm 1.83(3.36-9.47)$ & $5.2 \pm 2.02(1.24-10)$ & 0.7 \\
\hline PSA density $(\mathrm{ng} / \mathrm{mL} / \mathrm{cc})$ & $0.16 \pm 0.06(0.07-0.28)$ & $0.14 \pm 0.12(0.03-0.83)$ & $0.017^{*}$ \\
\hline TCIC (n) & $1.16 \pm 0.38(1-2)$ & $1.19 \pm 0.39(1-2)$ & 0.79 \\
\hline$\overline{\mathrm{TClQ}}(\mathrm{mm})$ & $1.88 \pm 1.36(1-6)$ & $1.96 \pm 1.34(0.1-7)$ & 0.64 \\
\hline TCIR (\%) & $15.56 \pm 11.93(5-50)$ & $15.31 \pm 12.81(0.55-50)$ & 0.75 \\
\hline
\end{tabular}


Table 5.

Number of patients managed with additional treatments in different PSA-density levels and grouping of patients with radical prostatectomy by ISUP grade.

\begin{tabular}{|c|c|c|c|}
\hline & & $\begin{array}{c}\text { PSA-D } \leq 0.13^{*}(n=58) \\
\text { Mean } \pm \text { SD }\end{array}$ & $\begin{array}{c}\text { PSA-D }>0.13^{*}(n=49) \\
\text { Mean } \pm \text { SD }\end{array}$ \\
\hline \multicolumn{2}{|l|}{ Age (years) } & $63.06 \pm 5.41$ & $62.93 \pm 6.39$ \\
\hline & & $\begin{array}{c}\text { ATR } \\
n=7(\%)\end{array}$ & $\begin{array}{c}\text { ATR } \\
n=17(\%)\end{array}$ \\
\hline Treated with radical prostatectomy & $\begin{array}{l}\text { ISUP } 1 \\
\text { ISUP } 2 \\
\text { ISUP } 3 \\
\text { ISUP } 4 \\
\text { ISUP } 5 \\
\text { pT0** }\end{array}$ & $\begin{array}{c}6(85.71) \\
0 \\
0 \\
0 \\
1(14.28) \\
0\end{array}$ & $\begin{array}{c}6(35.29) \\
5(29.41) \\
1(5.88) \\
0 \\
0 \\
1(5.88)\end{array}$ \\
\hline \multicolumn{2}{|l|}{ Treated with RT } & 0 & $2(11.76)$ \\
\hline \multicolumn{2}{|l|}{ Treated with ADT } & 0 & $2(11.76)$ \\
\hline
\end{tabular}

al treatment; in 49/107 patients PSA-D was more than $0.13 \mathrm{ng} / \mathrm{mL} / \mathrm{cc}$ and 17 (34.69\%) of them required additional treatment (Table 5). When the cut-off level of PSA-D was based on 0.13 $\mathrm{ng} / \mathrm{mL} / \mathrm{cc}$, the follow-up period until the decision to start additional treatment was 26.14 months for PSA-D $\leq 0.13$ and 25.4 months for PSA-D > 0.13) months, respectively.

Two patients with PSA-D > 0.13 $\mathrm{ng} / \mathrm{mL} / \mathrm{cc}$ lost their chance of definitive treatment. PSA-D was associated with predicting of additional treatment requirement for active surveillance $(\mathrm{p}=$ 0.017 ) and ROC analysis showed that the optimal threshold was $0.13 \mathrm{ng} / \mathrm{mL} / \mathrm{cc}$ (sensitivity: 70.8\%; specificity: 57.1\%). Twenty patients underwent radical prostatectomy (RP) as additional treatment in follow-up. Average follow-up

Table 6.

Number of PCa patients managed with active surveillance according to ISUP grades.

\begin{tabular}{|l|c|c|}
\hline ISUP Grades (PCa in RP) & $\mathbf{n}$ (Overall) & $\%$ \\
\hline ISUP 1 & 12 & 60 \\
\hline ISUP 2 & 5 & 25 \\
\hline ISUP 3 & 1 & 5 \\
\hline ISUP 5 & 1 & 5 \\
\hline pT0* & 1 & 5 \\
\hline $\begin{array}{l}\text { *: tumor tissue was not detected in pathology specimen after radical prostatectomy. } \\
\text { ISUP: International Society of Urologic Pathologists. PCa: Prostate cancer. }\end{array}$ \\
\hline
\end{tabular}

months (range, 12 to 134 months); tPSA changes in the following years after diagnosis were shown in Table 3. There was no difference in TCIC, TCIQ and TCIR levels between the 2 groups as reported in Table 4 ( $p>0.05)$. The PSA-D was less than $0.13 \mathrm{ng} / \mathrm{mL} / \mathrm{cc}$ in $58 / 107$ patients and only 7 (12.06\%) of them required addition- time until RP was 25.47 months (range, 12 to 60 months). In patients with prostate cancer managed by active surveillance, annual changes of tPSA levels in follow-up according to patients with PSA-D $>0.13$ or $0.13 \leq$ ng/mL/cc are shown in Figure 1.

Pathology results of radical prostatectomy specimens according to the ISUP classification are reported in Table 6. Twelve out of 20 (60\%) radical prostatectomy specimens were graded ISUP 1. Two patients preferred radiotherapy (RT) and another two received androgendeprivation therapy (RT not approved by radiation oncologist) as additional treatment (Figure 2).

Additional treatments were offered to other two patients, but the patients refused to additional treatments and were removed from the treatment protocol of their own volition. Additional cancers were observed in 3 of 107 patients under follow-up, one patient was diagnosed with bladder cancer and two patient diagnosed with renal cell cancer. Two patients died due to non-cancerous causes (chronic heart failure and lymphoma).

\section{Figure 1.}

In patients with prostate cancer managed by active surveillance. annual changes of PSA levels in follow-up according to patients with PSA-Density $>0.13$ or $0.13 \leq n g / m L / c c$.

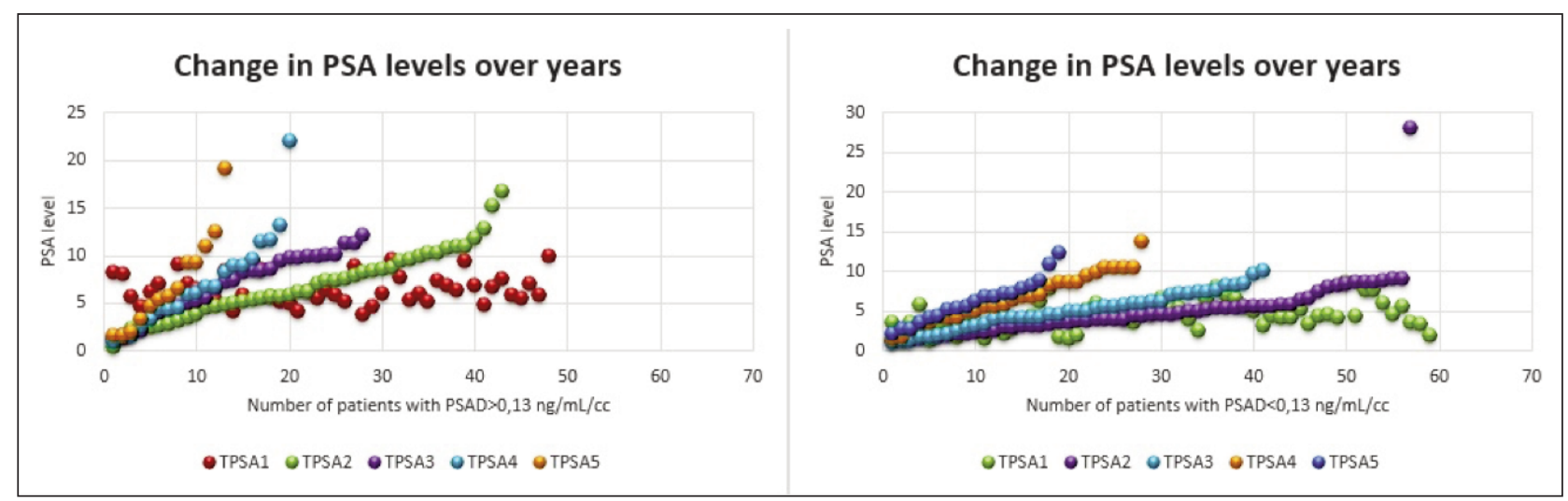

PSA: prostate specific antigen. PSAD: prostate-specific antigen density.

TPSA1.2.3.4.5: First, second, third, fourth and fifth year total prostate specific antigen levels in follow-up. 
Figure 2.

The flowchart includes patients who remained on surveillance or moved to active treatment (RP, RT or ADT) and annual change of tPSA for 5-year in the study population.

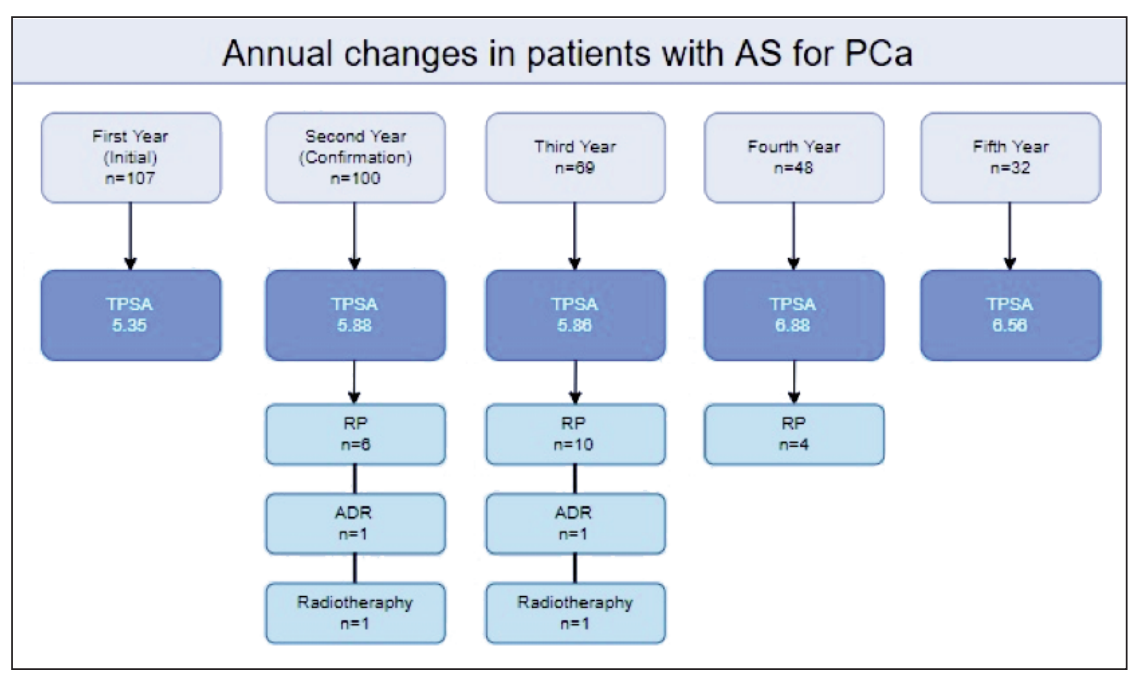

the patients needed additional treatment and follow-up period until the decision to start surgical or hormonal theraphy was shortened when the PSA-D was increased. In patients with PSA-D greater than $0.13 \mathrm{ng} / \mathrm{mL} / \mathrm{cc}$, high Gleason scores (from $3+4$ to $4+5$ ) were detected when radical prostatectomy was performed as definitive treatment. In a study by Camur et al., upgrading was seen in 35 (44.8\%) of 78 patients included in the study, but PV had no significant effect on upgrading in AS appropriate patients (16). Although PV is ineffective, AS criterias may include PSA-D. Patients with PSA-D levels $<0.07 \mathrm{ng} / \mathrm{mL} / \mathrm{cc}$ were not upgraded and needed no additional treatment. Especially very low PSA-D levels $(<0.07)$ are extremely safe. We think that risk classifications

\section{Discussion}

Active Surveillance is a well-recognized option in patients with low (clinical stage T1-T2a and tPSA $<10$ ng/mL and Grade Group 1) and very low-risk (clinical stage $\mathrm{T} 1-\mathrm{T} 2 \mathrm{a}$ and $\mathrm{tPSA}<10 \mathrm{ng} / \mathrm{mL}$ and Grade Group 1 and PSA-Density $<0.15 \mathrm{ng} / \mathrm{mL} / \mathrm{cc}$ and $<34 \%$ of biopsy cores positive and no core with $>50 \%$ involved) PCa (9). There are different protocols and selection criterias to select the appropriate patient. tPSA, clinical stage, and number of positive cores, each core involvement, PSA-D and a life expectancy of at least $10 \mathrm{yr}$ are the basis of different protocols.

Prostate specific antigen threshold values in AS are variable, but generally less than $10 \mathrm{ng} / \mathrm{mL}$, in a study conducted by Royal Marsden Clinic in 2008, tPSA threshold was determined $\leq 15 \mathrm{ng} / \mathrm{mL}$ (10). Clinical stage Tlc or $\mathrm{T} 2 \mathrm{a}$ is accepted as eligible for AS guidelines. The tPSA threshold values for AS suggested by the guidelines have a low risk potential. However, we think that the low Gleason score is more valuable than the low PSA, and the threshold value of high PSA values (above $10 \mathrm{ng} / \mathrm{mL}$ ) should be preferred in appropriate patients. In our clinical practice, we apply AS more flexibly in informed patients (information including detailed explanations about their condition and the likely outcomes of treatment) than the guidelines suggest.

PSA-Density is a predictor of upgrading of ISUP degree after radical prostatectomy and it is used safely in AS (11-13). The threshold value of PSA-D is $0.15 \mathrm{ng} / \mathrm{mL} / \mathrm{cc}$ in current guidelines, but nowadays, in some studies, the cut-off value of PSA-D of $0.08 \mathrm{ng} / \mathrm{mL} / \mathrm{cc}$ indicates significant risk for disease progression (14). Jin et al. found that optimal cut-off level of PSA-D was $0.13 \mathrm{ng} / \mathrm{mL} / \mathrm{cc}$ (11). Barayan et al. found that a PSA-D > $0.15 \mathrm{ng} / \mathrm{ml} / \mathrm{cc}$ is an important predictor for disease progression (15). In our cohort, PSA-D was the only factor that was statistically significant in predicting the need for additional treatment and optimal cut-off level was $0.13 \mathrm{ng} / \mathrm{mL} / \mathrm{cc}$. In patients with PSA-D > $0.13 \mathrm{ng} / \mathrm{mL} / \mathrm{cc}$, a larger part of according to PSA-D levels will contribute to the determination of secondary or tertiary biopsy requirements in follow-up protocols of patients and believe that the cutoff value of the recommended PSA-D in the guidelines should be updated to below $0.15 \mathrm{ng} / \mathrm{mL} / \mathrm{cc}$.

The number of positive cores is limited by two according to many authors. In California University, this value should not exceed 33\% of the total number of cores taken (17). Many guidelines and Authors have suggested that involvement rate in cores should be limited to 50\%. Soloway et al. suggested a rate of 20\% (18) and Porten et al. of 33\% (19). We think that $50 \%$ is an optimal value, but this rate cannot be evaluated in inadequate biopsy samples. The ideal core length should be more than $1.5 \mathrm{~cm}$.

Tumor measurements such as number of positive cores, fraction of positive cores, linear percentage of carcinoma in each core or across all sites and linear millimeters of carcinoma in each core or across all sites are used for patient selection in AS (20). The actual area of the tumor in millimeters is used to calculate percentage (21). The percent of needle biopsy cores and surface area are the strongest predictors of tumor volume and pathological stage (22). In the literature, many articles found that maximum percent core involvement at diagnosis was associated with progression (23-26). Sternberg et al. (27) and Iremashvili et al. (28) created nomograms that include number of positive cores and percent of positive cores at diagnosis. However, disease progression and extent of cancer on biopsy are not associated significantly in some studies $(29,30)$.

In this area; open to different interpretations, TCIC, TCIQ and TCIR measurements are optional and we thought that the TCIC, TCIQ and TCIR would be more significant than the amount of tumor rate in each core. Quintal et al found that TCIC and TCIR were significantly stronger than linear percentage of cancer or greatest millimeter length in each core to predict biochemical recurrence (31). Brimo et al. found that TCIR is closely associated with stage and biochemical failure (32). However, Park et al. found that 
these paramaters were not significant in predicting $\mathrm{pT} 3$ disease (33). Russo et al. found a cut-off value of $0.4 \mathrm{~mm}$ for each core (34). Today, clearly defined and accepted values are still not available to predict total tumor burden. In this study, we found that the measurements except than PSA-D were insufficient in predicting the need for additional treatment.

One of the most important points in AS is patient compliance to follow-up periods. Patients may want to withdraw from the AS protocol because they can not accept living with recurrent PSA measurements, examinations, biopsies and diagnosis of tumor. For this reason, followup of this protocol with non-invasive methods such as mpMRI is extremely valuable. Alberts et al found that Prostate Imaging Reporting and Data System score of 13 and PSA-D of $<0.15 \mathrm{ng} / \mathrm{mL} / \mathrm{cc}$ did not show Gleason score upgrading in biopsies at each time point of surveillance (35). AS may be more preferable to patients with low risk PCa due to reduced biopsy requirements in the future. Especially combination of PSA-D and mpMRI may be the future of this therapy management.

The limitations of the study are the evaluation of data retrospectively, the relatively patients' low compliance with the follow-up protocol, the comparatively short mean follow-up period and the lack of multiparametric MRI findings. More studies are needed to predict total tumor burden at diagnosis and additional treatment requirement over time.

\section{Conclusions}

Active surveillance in the treatment of prostate cancer is a proven protocol and there is a high degree of consensus on its criterias. Although TCIC, TCIQ and TCIR measurements are thought to help us to have an idea about the total tumor burden and progression, there is no contribution to predict the need for additional treatment. Therefore, we don't think they need to be measured routinely. Cut-off value of PSA-D as a 0.13 $\mathrm{ng} / \mathrm{mL} / \mathrm{cc}$ may be effective in determining the risk group and may be predictive of the need for additional treatment in the follow-up.

\section{ACKNOWLEDGEMENTS OF FINANCIAL SUPPORT}

The authors would like to thank the entire staff of the Department of Urology, Sultan Abdulhamid Han Education and Research Hospital and certify that there is no conflict of interest with any financial organization regarding the material discussed in the manuscript.

\section{REFERENCES}

1. Siegel RL, Miller KD, Jemal A. Cancer statistics, 2018. CA Cancer J Clin 2018; 68:7-30.

2. Jemal A, Siegel R, Ward E, et al. Cancer statistics, 2007. CA Cancer J Clin. 2007; 57:43-66.

3. Klotz L. Active surveillance with selective delayed intervention: a biologically nuanced approach to favorable-risk prostate cancer. Clin Prostate Cancer. 2003; 2:106-110.

4. Koo KC, Lee KS, Jeong JY, et al. Pathological and oncological fea- tures of Korean prostate cancer patients eligible for active surveillance: analysis from the K-CaP registry. Jpn J Clin Oncol. 2017; 47:981-985.

5. Lee DH, Koo KC, Lee SH, et al. Low-risk prostate cancer patients without visible tumor (T1C) on multiparametric MRI could qualify for active surveillance candidate even if they did not meet inclusion criteria of active surveillance protocol. Jpn J Clin Oncol. 2013; 43:553-558.

6. Corcoran AT, Peele PB, Benoit RM. Cost comparison between watchful waiting with active surveillance and active treatment of clinically localized prostate cancer. Urology. 2010; 76:703-707.

7. Egevad L, Delahunt B, Srigley JR, et al. International Society of Urological Pathology (ISUP) grading of prostate cancer - An ISUP consensus on contemporary grading. APMIS. 2016; 124:433-435.

8. Epstein JI, Allsbrook WC Jr., Amin MB, et al. The 2005 International Society of Urological Pathology (ISUP) Consensus Conference on Gleason Grading of Prostatic Carcinoma. Am J Surg Pathol. 2005; 29:1228-1242.

9. Thostrup M, Thomsen FB, Iversen P, et al. Active surveillance for localized prostate cancer: update of a prospective single-center cohort. Scand J Urol. 2018; 52:14-19.

10. van As NJ, Norman AR, Thomas K, et al. Predicting the probability of deferred radical treatment for localised prostate cancer managed by active surveillance. Eur Urol. 2008; 54:1297-1305.

11. Jin BS, Kang SH, Kim DY, et al. Pathological upgrading in prostate cancer patients eligible for active surveillance: Does prostate-specific antigen density matter? Korean J Urol. 2015; 56:624-629.

12. Kotb AF, Tanguay S, Luz MA, et al. Relationship between initial PSA density with future PSA kinetics and repeat biopsies in men with prostate cancer on active surveillance. Prostate Cancer Prostatic Dis. 2011; 14:53-57.

13. Loeb S, Bruinsma SM, Nicholson J, et al. Active surveillance for prostate cancer: a systematic review of clinicopathologic variables and biomarkers for risk stratification. Eur Urol. 2015; 67:619-626.

14. San Francisco IF, Werner L, Regan MM, et al. Risk stratification and validation of prostate specific antigen density as independent predictor of progression in men with low risk prostate cancer during active surveillance. J Urol. 2011; 185:471-476.

15. Barayan GA, Brimo F, Begin LR, et al. Factors influencing disease progression of prostate cancer under active surveillance: a McGill University Health Center cohort. BJU Int. 2014; 114:E99-E104.

16. Camur E, Coskun A, Kavukoglu, et al. Prostate volume effect on Gleason score upgrading in active surveillance appropriate patients. Arch Ital Urol Androl. 2019; 91:93-96

17. Cooperberg MR, Cowan JE, Hilton JF, et al. Outcomes of active surveillance for men with intermediate-risk prostate cancer. J Clin Oncol. 2011; 29:228-234.

18. Soloway MS, Soloway CT, Eldefrawy A, et al. Careful selection and close monitoring of low-risk prostate cancer patients on active surveillance minimizes the need for treatment. Eur Urol. 2010; 58:831-835.

19. Porten SP, Whitson JM, Cowan JE, et al. Changes in prostate cancer grade on serial biopsy in men undergoing active surveillance. J Clin Oncol. 2011; 29:2795-2800.

20. Amin MB, Lin DW, Gore JL, et al. The critical role of the pathologist in determining eligibility for active surveillance as a management option in patients with prostate cancer: consensus statement with recommendations supported by the College of American Pathologists, International Society of Urological Pathology, Association of Directors 
of Anatomic and Surgical Pathology, the New Zealand Society of Pathologists, and the Prostate Cancer Foundation. Arch Pathol Lab Med. 2014; 138:1387-1405.

21. Draisma G, Boer R, Otto SJ, et al. Lead times and overdetection due to prostate-specific antigen screening: estimates from the European Randomized Study of Screening for Prostate Cancer. J Natl Cancer Inst. 2003; 95:868-878.

22. Sebo TJ, Bock BJ, Cheville JC, et al. The percent of cores positive for cancer in prostate needle biopsy specimens is strongly predictive of tumor stage and volume at radical prostatectomy. J Urol. 2000; 163:174-178.

23. Tseng KS, Landis P, Epstein JI, et al. Risk stratification of men choosing surveillance for low risk prostate cancer. J Urol. 2010; 183:1779-1785.

24. Bul M, Zhu X, Valdagni R, et al. Active surveillance for low-risk prostate cancer worldwide: the PRIAS study. Eur Urol. 2013; 63:597-603.

25. Eggener SE, Mueller A, Berglund RK, et al. A multi-institutional evaluation of active surveillance for low risk prostate cancer. J Urol. 2013; 189:S19-25.

26. Venkitaraman $R$, Norman $A$, Woode-Amissah $R$, et al. Predictors of histological disease progression in untreated, localized prostate cancer. J Urol. 2007; 178:833-837.

27. Sternberg IA, Yu C, Keren Paz GE, et al. Predicting progression in patients followed with active surveillance for low-risk prostate cancer. American Society of Clinical Oncology; 2014.

28. Iremashvili V, Burdick-Will J, Soloway MS. Improving risk stratification in patients with prostate cancer managed by active surveillance: a nomogram predicting the risk of biopsy progression. BJU Int. 2013; 112:39-44.
29. Cary KC, Cowan JE, Sanford M, et al. Predictors of pathologic progression on biopsy among men on active surveillance for localized prostate cancer: the value of the pattern of surveillance biopsies. Eur Urol. 2014; 66:337-342.

30. Adamy A, Yee DS, Matsushita K, et al. Role of prostate specific antigen and immediate confirmatory biopsy in predicting progression during active surveillance for low risk prostate cancer. J Urol. 2011; 185:477-482.

31. Quintal MM, Meirelles LR, Freitas LL, et al. Various morphometric measurements of cancer extent on needle prostatic biopsies: which is predictive of pathologic stage and biochemical recurrence following radical prostatectomy? Int Urol Nephrol. 2011; 43:697-705.

32. Brimo F, Vollmer RT, Corcos J, et al. Prognostic value of various morphometric measurements of tumour extent in prostate needle core tissue. Histopathology. 2008; 53:177-183.

33. Park EA, Lee HJ, Kim KG, et al. Prediction of pathological stages before prostatectomy in prostate cancer patients: analysis of 12 systematic prostate needle biopsy specimens. Int J Urol. 2007; 14:704-708.

34. Russo GI, Cimino S, Castelli T, et al. Percentage of cancer involvement in positive cores can predict unfavorable disease in men with low-risk prostate cancer but eligible for the prostate cancer international: active surveillance criteria. Urol Oncol. 2014; 32:291-296.

35. Alberts AR, Roobol MJ, Drost FH, et al. Risk-stratification based on magnetic resonance imaging and prostate-specific antigen density may reduce unnecessary follow-up biopsy procedures in men on active surveillance for low-risk prostate cancer. BJU Int. 2017; 120:511-519.

\section{Correspondence}

Caner Ediz, MD (Corresponding Author)

drcanerediz@gmail.com

Serkan Akan, MD

drserkanakan@hotmail.com

Muhammed Cihan Temel, MD

dr.cihantemel@gmail.com

Omer Yilmaz, MD

dr_omeryilmaz@yahoo.com

Department of Urology, Sultan Abdulhamid Han Education and Research Hospital, Istanbul, Turkey

Tibbiye Street. Selimiye neighborhood. Uskudar/Istanbul 